\title{
The Application of Computer Software Development Technology in Medical Institutions
}

\section{Hanbing Chu}

Information Engineering Institute, Zhengzhou University of Industrial Technology, Xinzheng, China, 451150

Keywords: computer; software development technology; application

\begin{abstract}
With the rapid development of modern information technology, computer software has been widely used and plays a very important role in all walks of life. Nowadays, with the improvement of intelligence and information technology, the value of computer software development technology is becoming more and more prominent. Only by constantly innovating and reforming the computer software development technology, can the computer industry continue to develop healthily and its related industries continue to mature. This paper mainly analyzes the application of computer software development technology in practice.
\end{abstract}

\section{Introduction}

The development of computer plays an important role in promoting social progress, and is also related to the improvement of people's living standard. At present, computers are very important in the fields of medical, economic management and education in China. Computer software development technology is the key to computer development, and also an important support for China's economic development. Therefore, it is of great significance to analyze and study the application of computer software development technology and its future development trend.

\section{The Application of Computer Software Development Technology in Medical Institutions}

Computer software development technology is widely used in medical field. By introducing advanced computer software development technology into medical institutions, it can effectively promote the development of medical technology. At the same time, it can simplify the working process, improve the management quality and level of the hospital, improve the accuracy of doctors' diagnosis and improve the work efficiency.

The hospital information management system is established by the application of computer software development technology. Its basic function is to realize the computer management of the main business work of the hospital. Thus, it can improve the working efficiency, plug the loopholes in management, realize the service tenet of the patient as the center, solve the many problems that have plagued the hospital for a long time, and improve the service quality comprehensively. Hospital information management system provide the information needed to diagnosis, treatment and nursing process in time and accurately for doctors, nurses and other health care professionals improve the quality of treatment and nursing service, at the same time reduce the treatment process, shorten the treatment time, reduce queuing phenomenon.

\section{The Application of Door Emergency Registration System}

With the rapid development of economy, people pay more and more attention to their health, so more and more people go to the hospital. Registration which is difficult and disorder is one of the urgent problems that hospitals need to solve. The problem of registration is analyzed systematically and comprehensively by using computer software development technology, and a targeted and complete computer registration system is worked out. Through this system, the patient can understand the main working hours and the diagnosis direction of different doctors in different 
departments of the hospital anytime and anywhere, greatly facilitated the patient's practice, and thus greatly improved the efficiency of the hospital registration. Using computer software development technology to establish outpatient files, the hospital carries out real name IC card for medical interrogation, and patients can also deposit advance payment. The application of this system can also deal with outpatient registration, back number, appointment registration and transfer room etc. The system also supports multiple forms of payment, including public, private and health care. The registration fee information can also be checked by the registration form, the registration branch and the branch statistics retrieval.

\section{The Application of Medical Record Information Management}

In the information management of hospital medical records, the patient's information and treatment plan should be recorded. In the past, the hospital used artificial paper mode to record and record, not only to spend a lot of time and energy, but also very cumbersome in management, and easy to make mistakes. This method can not meet the needs of modern social development, nor can it meet the development needs of hospitals. By introducing the computer software development technology into the medical institution and developing the office software of the hospital, it is timely, effective and accurate to record the patient's various treatment information and save it. At the same time, the office software can simplify the tedious work, reduce the labor intensity of the staff of the hospital, relieve the staff of the hospital from the tedious work, and improve the work efficiency and quality. The application of computer software development technology in hospital medical and health environment has greatly improved its therapeutic efficiency and made important contribution to human health.

\section{The Application of Medical Equipment Management}

Bultrasound is a very common medical method in medical and health institutions. It is the use of computer software development technology to achieve patient examination of image display. B ultrasound is used to check people's health condition by ultrasonic technology. According to the ultrasound scan, the doctor can analyze the patient's condition effectively, accurately grasp the patient's condition, and do not mislead the treatment. At the same time, computer software development technology can also be used in CT medical equipment, X-ray machine, analyzer and other equipment. It can be said that computer software development technology plays an important role in promoting the progress and development of modern medical technology and realizing efficient and scientific medical diagnosis.

\section{The Application of the Dean's Query System}

Using computer software to develop the dean's query system, combined with the actual situation of the hospital, in which set up some functional modules in the dean's inquiry system, such as financial status, medical dynamics, drug information, patient flow statistics, patient information, etc. The director of the hospital can provide comprehensive quantitative analysis information on the basis of the comprehensive and timely understanding of hospital management and working conditions. It can realize the classification and processing of the data produced or collected by other applications of the hospital information management system, which conforms to the business rules, and then provides a number of reference data for the decision of the president and the understanding of the work situation in the hospital. The hospital dean inquisition system and outpatient management system, hospital management system, pharmacy management system and other modules implement data sharing, so that they can coordinate and unify.

\section{Conclusion}

Above all, by analyzing the application of computer software development technology, we have 
a deeper understanding of computer software development. It is recognized that the development of software must be based on the actual application needs of enterprises and users. At the same time, software can play an important role and value in enterprises and users. The development of computer software development technology is a process of real-time updating. It is necessary to take full consideration of the future development trend and take appropriate ways to improve the efficiency and effect of software development, enhance its application and realize the smooth and benign development of computer software development.

\section{References}

[1] Dai Tianfeng. Application and development trend of computer software development technology [J]. Modern industrial economy and information,2017, (20):64-66.

[2] Fan Wei. Application and development trend of computer software development technology in the new era [J]. Computer optical disk software and application,2016,17(13):80+82.

[3] Guo Changjiang. Application and development trend of computer software development technology in the new era [J]. Communication world,2017, (02):110.

[4] Chen Ke, Wang Zhihai, Yin Hongfeng. Application and development trend of computer software development technology in the new era [J]. China strategic emerging industry,2017, (32):91.

[5] Zhang Dongxia, Miao Xin, Liu Liping, Zhang Yan, Liu Keyan. Research on the development of big data technology of smart power grid [J]. Journal of Chinese electrical engineering, 2015,35 (01):2-12. 